\title{
The $m$-Cover Posets and the Strip-Decomposition of m-Dyck Paths
} (Extended Abstract)

\author{
Myrto Kallipoliti and Henri Mühle $\rrbracket^{*}$
}

Fakultät für Mathematik, Universität Wien.

\begin{abstract}
In the first part of this article we present a realization of the $m$-Tamari lattice $\mathcal{T}_{n}^{(m)}$ in terms of $m$-tuples of Dyck paths of height $n$, equipped with componentwise rotation order. For that, we define the $m$-cover poset $\mathcal{P}^{\langle m\rangle}$ of an arbitrary bounded poset $\mathcal{P}$, and show that the smallest lattice completion of the $m$-cover poset of the Tamari lattice $\mathcal{T}_{n}$ is isomorphic to the $m$-Tamari lattice $\mathcal{T}_{n}^{(m)}$. A crucial tool for the proof of this isomorphism is a decomposition of $m$-Dyck paths into $m$-tuples of classical Dyck paths, which we call the strip-decomposition. Subsequently, we characterize the cases where the $m$-cover poset of an arbitrary poset is a lattice. Finally, we show that the $m$-cover poset of the Cambrian lattice of the dihedral group is a trim lattice with cardinality equal to the generalized FussCatalan number of the dihedral group.

Résumé. Dans la première partie de cet article nous présentons une réalisation du treillis $m$-Tamari $\mathcal{T}_{n}^{(m)}$ à l'aide de $m$-uplets de chemins de Dyck de hauteur $n$, équipés de l'ordre de rotation composante par composante. Pour cela, nous définissons le poset de $m$-couverture $\mathcal{P}^{\langle m\rangle}$ d'un poset borné quelconque $\mathcal{P}$, et montrons que la plus petite complétion en treillis du poset de $m$-couverture du treillis de Tamari $\mathcal{T}_{n}$ est isomorphe au treillis $m$-Tamari $\mathcal{T}_{n}^{(m)}$. Un outil crucial pour la preuve de cet isomorphisme est une décomposition des chemins $m$-Dyck en $m$-uplets de chemins de Dyck usuels, que nous appelons la décomposition en bandes. Par la suite, nous caractérisons les cas où le poset de $m$-couverture d'un poset donné est un treillis. Enfin nous montrons que le poset de $m$-couverture du treillis Cambrien du groupe diédral est un treillis svelte de cardinalité le nombre généralisé de Fuss-Catalan du groupe diédral.
\end{abstract}

Keywords: m-Tamari lattice, m-Dyck paths, m-cover poset, Fuss-Catalan combinatorics, Symmetric group, Dihedral group, Left-modularity, Trimness, Möbius function

\section{Introduction}

The Tamari lattices $\mathcal{T}_{n}$ were originally defined in [11] as a partial order on bracketings of strings and constitute a well-studied member of the large group of Catalan objects, i.e. families of mathematical

\footnotetext{
*E-mail: myrto.kallipoliti, henri.muehle\}@univie.ac.at. The authors were supported by the FWF research grant no. Z130-N13.
}

1365-8050 @ 2014 Discrete Mathematics and Theoretical Computer Science (DMTCS), Nancy, France 
objects such that the cardinality of their $n$-th member is given by the $n$-th Catalan number, defined by

$$
\operatorname{Cat}(n)=\frac{1}{n+1}\left(\begin{array}{c}
2 n \\
n
\end{array}\right)
$$

The realization of $\mathcal{T}_{n}$ as a poset on Dyck paths, led Bergeron and Préville-Ratelle to the definition of so-called $m$-Tamari lattices $\mathcal{T}_{n}^{(m)}$, by using $m$-Dyck paths and a straightforward generalization of the rotation order, see [1, Section 5]. In the case $m=1$, the $m$-Tamari lattices reduce to the classical Tamari lattices, and the cardinality of $\mathcal{T}_{n}^{(m)}$ is the well-known Fuss-Catalan number, defined by

$$
\mathrm{Cat}^{(m)}(n)=\frac{1}{m n+1}\left(\begin{array}{c}
(m+1) n \\
n
\end{array}\right) .
$$

In the present paper we show that $\mathcal{T}_{n}^{(m)}$ can be realized in terms of $m$-tuples of Dyck-paths equipped with componentwise rotation order. In order to achieve this goal, we introduce two constructions: on the one hand, given an arbitrary bounded poset $\mathcal{P}$ we define a certain subposet of the $m$-fold direct product of $\mathcal{P}$ with itself, which we call the $m$-cover poset of $\mathcal{P}$, and denote it by $\mathcal{P}^{\langle m\rangle}$; on the other hand, we provide a new decomposition of $m$-Dyck paths of height $n$ into $m$-tuples of classical Dyck paths of height $n$, which we call the strip-decomposition. We proceed by showing that the Dedekind-MacNeille completion of $\mathcal{T}_{n}^{\langle m\rangle}$ - which is the smallest lattice containing $\mathcal{T}_{n}^{\langle m\rangle}$ —is isomorphic to $\mathcal{T}_{n}^{(m)}$ (i) More precisely, we prove the following theorem.

Theorem 1.1 For $m, n>0$ we have $\mathcal{T}_{n}^{(m)} \cong \mathbf{D M}\left(\mathcal{T}_{n}^{\langle m\rangle}\right)$, where $\mathbf{D M}$ denotes the Dedekind-MacNeille completion.

We conjecture that there is a more explicit way to realize $\mathcal{T}_{n}^{(m)}$ as a lattice of $m$-tuples of Dyck paths: if we modify the strip-decomposition of the $m$-Dyck paths of height $n$ in a certain way-a procedure we call bouncing - then we obtain a slightly different set of $m$-tuples of Dyck paths, and computer experiments suggest that this set under componentwise rotation order is isomorphic to $\mathcal{T}_{n}^{(m)}$, see Remark 4.5 below for the details.

Subsequently, we investigate properties of the $m$-cover poset and prove the following theorem which characterizes the cases where this poset is a lattice ${ }^{(i i)}$

Theorem 1.2 Let $\mathcal{P}$ be a bounded poset, with least element $0_{\mathcal{P}}$ and greatest element $1_{\mathcal{P}}$, and let $m>1$. Then, the $m$-cover poset $\mathcal{P}^{\langle m\rangle}$ is a lattice if and only if the Hasse diagram of $\mathcal{P}$ with $0_{\mathcal{P}}$ removed is a tree rooted at $1_{\mathcal{P}}$.

Finally, we consider the Cambrian lattice $\mathcal{C}_{k}$ associated with the dihedral group $\mathfrak{D}_{k}$, see for instance [9]. It follows from Theorem 1.2 that its $m$-cover poset, $\mathcal{C}_{k}^{\langle m\rangle}$, is a lattice. In addition we prove that $\mathcal{C}_{k}^{\langle m\rangle}$ is trim, thus EL-shellable, we compute its cardinality and show that its Möbius function takes only values in $\{-1,0,1\}$, generalizing the properties of $\mathcal{C}_{k}$. In particular, we have the following result. (Recall that a closed interval $[x, y]$ in a lattice is called nuclear if $y$ is the join of atoms of $[x, y]$.)

(i) Notice the different superscripts!

(ii) In $[6$ Theorem 1.3] we refine Theorem 1.2 by characterizing in addition the cases where the lattice $\mathcal{P}\langle m\rangle$ is left-modular, or EL-shellable. 
Theorem 1.3 For $k>1$ and $m>0$, the poset $\mathcal{C}_{k}^{\langle m\rangle}$ is a trim lattice, and its cardinality is $\left(\begin{array}{c}m+1 \\ 2\end{array}\right) k+m+1$, which is the generalized Fuss-Catalan number of $\mathfrak{D}_{k}$. Furthermore, if $\mu$ denotes the Möbius function of $\mathcal{C}_{k}^{\langle m\rangle}$, then for $\mathbf{x}, \mathbf{x}^{\prime} \in C_{k}^{\langle m\rangle}$, with $\mathbf{x} \leq_{k} \mathbf{x}^{\prime}$, we have

$$
\mu\left(\mathbf{x}, \mathbf{x}^{\prime}\right)= \begin{cases}1, & \text { if }\left[\mathbf{x}, \mathbf{x}^{\prime}\right] \text { is nuclear and has two atoms } \\ -1, & \text { if } \mathbf{x}^{\prime} \text { covers } \mathbf{x}, \quad \text { or } \\ 0, & \text { otherwise }\end{cases}
$$

We note that the Tamari lattice $\mathcal{T}_{n}$ is also a special instance of a Cambrian lattice, see for example [8. Theorem 6.4]. Thus, the $m$-cover poset yields a Fuss-Catalan generalization of the above mentioned Cambrian lattices, namely a family of lattices parametrized by an integer $m$, such that the case $m=1$ yields the corresponding Cambrian lattice, and the cardinality of these lattices is the generalized FussCatalan number of the dihedral group and the symmetric group, respectively. However, for other Cambrian lattices, the $m$-cover poset or its lattice completion does not produce a poset having Fuss-Catalan-many elements. We note that a Fuss-Catalan generalization of the Cambrian lattices has recently been found by Stump, Thomas and Williams and will be available in a forthcoming paper. However, the lattice obtained for the dihedral group in their construction is different from $\mathcal{C}_{k}^{\langle m\rangle}$, and the lattice $\mathcal{T}_{n}^{(m)}$ is not recovered as a special case.

This article is organized as follows. In Section 2, we give the necessary order-theoretic notions and recall the definition of $m$-Dyck paths and the $m$-Tamari lattices, as well as some of their properties. In Section 3 we introduce our main constructions. In particular, in Section 3.1 we define the $m$-cover poset of an arbitrary bounded poset, while Section 3.2 is dedicated to the strip-decomposition of $m$-Dyck paths. Theorem 1.1 is then proved in Section 4 and the paper is completed with Section 5, where we prove Theorems 1.2 and 1.3

This work is an extended abstract of [6], and we have thus omitted most of the proofs which can be found in the detailed version of this abstract.

\section{Preliminaries}

In this section we recall the necessary notions which we will use in this article.

\subsection{Partially Ordered Sets}

Let $\mathcal{P}=\left(P, \leq_{\mathcal{P}}\right)$ be a finite partially ordered set (poset for short). We say that $\mathcal{P}$ is bounded if it has a least and a greatest element, denoted by $0_{\mathcal{P}}$ and $1_{\mathcal{P}}$, respectively, and its proper part is the poset obtained by removing the elements $0_{\mathcal{P}}$ and $1_{\mathcal{P}}$. We say that $\mathcal{P}$ is a lattice if for every two elements $x, y \in P$ there exists a least upper bound, which is called the join of $x$ and $y$ and is denoted by $x \vee_{\mathcal{P}} y$, and there exists a greatest lower bound, which is called the meet of $x$ and $y$ and is denoted by $x \wedge_{\mathcal{P}} y$. If $x<_{\mathcal{P}} y$ and there does not exist an element $z \in P$ with $x<_{\mathcal{P}} z<_{\mathcal{P}} y$, then we say that $y$ covers $x$, and we denote it by $x \lessdot_{\mathcal{P}} y$. In this case, we also say that $x$ is a lower cover of $y$ and that $y$ is an upper cover of $x$. By abuse of notation, we call an element $p \in P$ join-irreducible if it is not minimal, and has a unique lower cover, denoted by $p_{\star}$, and we write $\mathcal{J}(\mathcal{P})$ for the set of join-irreducible elements of $\mathcal{P}$. Similarly, we call p meet-irreducible if it is not maximal, and has a unique upper cover, denoted by $p^{\star}$, and we write $\mathcal{M}(\mathcal{P})$ for the set of meet-irreducible elements of $\mathcal{P}$. Moreover, we recall that given two posets The length of $\mathcal{P}$, 
denoted by $\ell(\mathcal{P})$, is the maximal length of a saturated chain from $0_{\mathcal{P}}$ to $1_{\mathcal{P}}$. Then, $\mathcal{P}$ is called extremal if $|\mathcal{J}(\mathcal{P})|=\ell(\mathcal{P})=|\mathcal{M}(\mathcal{P})|$, see [7]. If $\mathcal{P}$ is a lattice, then an element $x \in P$ is called left-modular if for every $y<\mathcal{P} z$ we have

$$
\left(y \vee_{\mathcal{P}} x\right) \wedge_{\mathcal{P}} z=y \vee_{\mathcal{P}}\left(x \wedge_{\mathcal{P}} z\right)
$$

If there exists a maximal chain consisting of left-modular elements, then $\mathcal{P}$ is called left-modular. If $\mathcal{P}$ is extremal and left-modular, then $\mathcal{P}$ is called trim, see [12]. For a detailed introduction on posets and lattices, we refer to [3, 10].

\subsection{The $m$-Dyck Paths and the $m$-Tamari Lattices}

The $m$-Dyck Paths. For $m, n \in \mathbb{N}$, we say that an $m$-Dyck path of height $n$ is a lattice path from $(0,0)$ to $(m n, n)$ which stays weakly above the line $x=m y$, and which consists only of up-steps and right-steps. Let $\mathcal{D}_{n}^{(m)}$ denote the set of all $m$-Dyck paths of height $n$. The cardinality of $\mathcal{D}_{n}^{(m)}$ is given by the Fuss-Catalan number defined in [2], see [4], Section 6].

Let $\mathfrak{p}$ be an $m$-Dyck path $\mathfrak{p} \in \mathcal{D}_{n}^{(m)}$. Then $\mathfrak{p}$ can be encoded by a sequence $\mathbf{u}_{\mathfrak{p}}=\left(u_{1}, u_{2}, \ldots, u_{n}\right)$, the so-called step sequence of $\mathfrak{p}$, which satisfies

$$
\begin{aligned}
& u_{1} \leq u_{2} \leq \cdots \leq u_{n}, \quad \text { and } \\
& u_{k} \leq m(k-1), \quad \text { for all } k \in\{1,2, \ldots, n\} .
\end{aligned}
$$

In other words, the entry $u_{k}$ represents at which $x$-coordinate the $k$-th up-step takes place. Equivalently, $\mathfrak{p}$ can also be encoded by a sequence $\mathbf{h}_{\mathfrak{p}}=\left(h_{1}, h_{2}, \ldots, h_{m n}\right)$, the so-called height sequence of $\mathfrak{p}$, satisfying

$$
\begin{aligned}
& h_{1} \leq h_{2} \leq \cdots \leq h_{m n} \leq n, \quad \text { and } \\
& h_{k} \geq\left\lceil\frac{k}{m}\right\rceil, \quad \text { for all } k \in\{1,2, \ldots, m n\} .
\end{aligned}
$$

In other words, the entry $h_{k}$ represents which height the path $\mathfrak{p}$ has at $x$-coordinate $k-\frac{1}{2}$. It is straightforward to prove the following conversion between the two sequences.

$$
\begin{aligned}
h_{k} & =\max \left\{j \in\{1,2, \ldots, n\} \mid u_{j}<k\right\}, & & \text { for all } k \in\{1,2, \ldots, m n\}, \quad \text { and } \\
u_{h_{k}+1} & =u_{h_{k}+2}=\cdots=u_{h_{k+1}}=k, & & \text { for all } k \in\{0,1, \ldots, m n\} \text { with } h_{k}<h_{k+1},
\end{aligned}
$$

where we set $h_{0}=0$.

Example 2.1 Let $\mathfrak{p} \in \mathcal{D}_{5}^{(3)}$ be the path shown in Figure $1(a)$ Its step sequence is $\mathbf{u}_{\mathfrak{p}}=(0,2,2,8,10)$, and its height sequence is $\mathbf{h}_{\mathfrak{p}}=(1,1,3,3,3,3,3,3,4,4,5,5,5,5,5)$.

The $m$-Tamari Lattices. Let $\mathfrak{p} \in \mathcal{D}_{n}^{(m)}$ with associated step sequence $\mathbf{u}_{\mathfrak{p}}=\left(u_{1}, u_{2}, \ldots, u_{n}\right)$. For $i \in\{1,2, \ldots, n\}$, we say the primitive subsequence of $\mathbf{u}_{\mathfrak{p}}$ at position $i$ is the unique subsequence $\left(u_{i}, u_{i+1}, \ldots, u_{k}\right)$ which satisfies

$$
\begin{aligned}
& u_{j}-u_{i}<m(j-i), \quad \text { for } j \in\{i+1, i+2, \ldots, k\}, \quad \text { and } \\
& \text { either } k=n, \quad \text { or } \quad u_{k+1}-u_{i} \geq m(k+1-i) .
\end{aligned}
$$




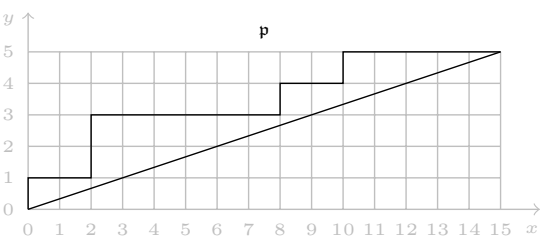

(a) A 3-Dyck path $\mathfrak{p}$ of height 5 .
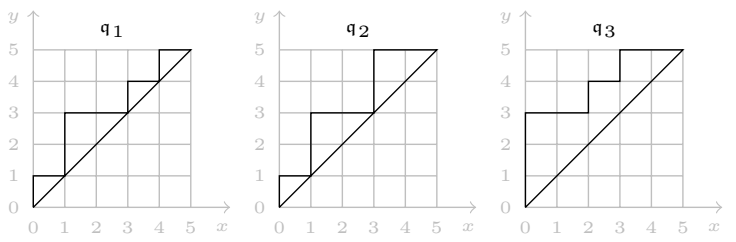

(b) The strip-decomposition of $\mathfrak{p}$.

Fig. 1: Illustration of the strip-decomposition.

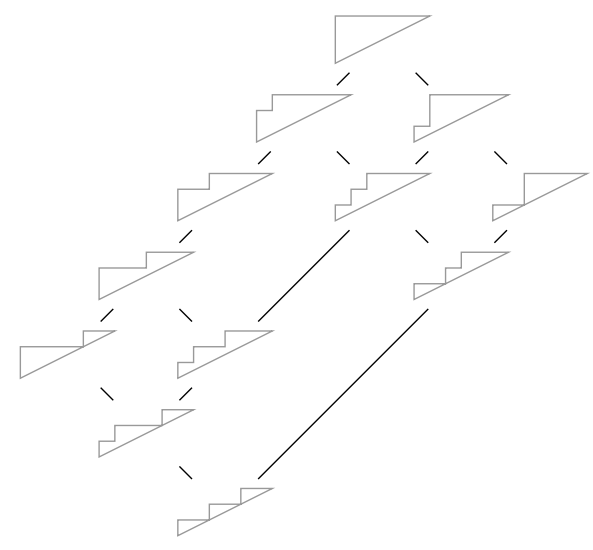

(a) The lattice $\mathcal{T}_{3}^{(2)}$.

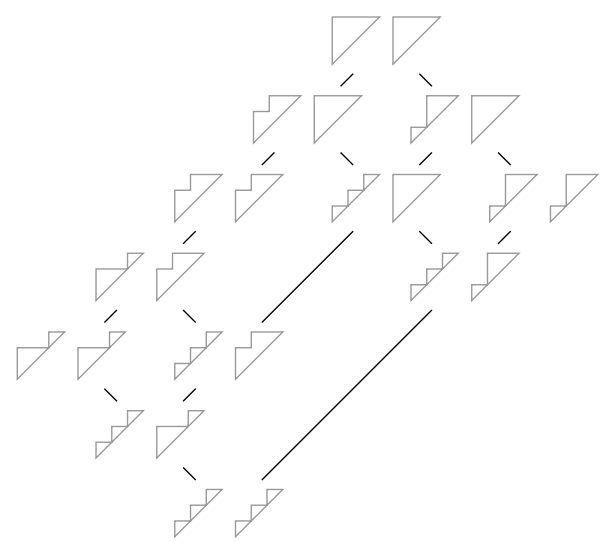

(b) The lattice $\mathcal{T}_{3}^{\langle 2\rangle}$.

Fig. 2: The 2-Tamari lattice of height 3.

Now, Bergeron and Préville-Ratelle define in [1. Section 5] a partial order on $\mathcal{D}_{n}^{(m)}$ as follows: let $\mathfrak{p}, \mathfrak{p}^{\prime} \in$ $\mathcal{D}_{n}^{(m)}$ such that $\mathbf{u}_{\mathfrak{p}}=\left(u_{1}, u_{2}, \ldots, u_{n}\right)$ is the step sequence of $\mathfrak{p}$, and $\mathbf{u}_{\mathfrak{p}^{\prime}}$ denotes the step sequence of $\mathfrak{p}^{\prime}$. Then, define

$$
\mathfrak{p} \lessdot_{R} \mathfrak{p}^{\prime} \quad \text { if and only if } \quad \mathbf{u}_{\mathfrak{p}^{\prime}}=\left(u_{1}, \ldots, u_{i}, u_{i+1}-1, \ldots, u_{k}-1, u_{k+1}, \ldots, u_{n}\right) \text {, }
$$

for some $i \in\{1,2, \ldots, n-1\}$ satisfying $u_{i}<u_{i+1}$ such that $\left\{u_{i+1}, u_{i+2}, \ldots, u_{k}\right\}$ is the primitive subsequence of $\mathbf{u}_{\mathfrak{p}}$ at position $i+1$. Let $\leq_{R}$ denote the transitive and reflexive closure of $\lessdot_{R}$, and call this partial order the rotation order on $\mathcal{D}_{n}^{(m)}$. Then, it is the statement of [2, Proposition 4] that $\mathcal{T}_{n}^{(m)}=\left(\mathcal{D}_{n}^{(m)}, \leq_{R}\right)$ is an interval in the classical Tamari lattice $\mathcal{T}_{m n}$, and is thus called the $m$-Tamari lattice of parameter $n$. Figure 2(a) shows the 2-Tamari lattice $\mathcal{T}_{3}^{(2)}$.

In the following two propositions, the proofs of which can be found in [6], we characterize the joinand meet-irreducible elements of $\mathcal{T}_{n}^{(m)}$.

Proposition 2.2 An element $\mathfrak{p} \in \mathcal{D}_{n}^{(m)}$ is meet-irreducible in $\mathcal{T}_{n}^{(m)}$ if and only if its step sequence $\mathbf{u}_{\mathfrak{p}}=$ 
$\left(u_{1}, u_{2}, \ldots, u_{n}\right)$ satisfies

$$
u_{j}= \begin{cases}0, & \text { for } j \leq i \\ a, & \text { for } j>i\end{cases}
$$

where $1 \leq a \leq$ mi and $i \in\{1,2, \ldots, n-1\}$.

Proposition 2.3 An element $\mathfrak{p} \in \mathcal{D}_{n}^{(m)}$ is join-irreducible in $\mathcal{T}_{n}^{(m)}$ if and only if its step sequence $\mathbf{u}_{\mathfrak{p}}=$ $\left(u_{1}, u_{2}, \ldots, u_{n}\right)$ satisfies

$$
u_{j}= \begin{cases}m(j-1), & \text { for } j \notin\{i, i+1, \ldots, k\}, \\ m(j-1)-s, & \text { for } j \in\{i, i+1, \ldots, k\}\end{cases}
$$

for exactly one $i \in\{1,2, \ldots, n\}$, where $k \in\{i, i+1, \ldots, n\}$ and $s \in\{1,2, \ldots, m\}$.

\section{The Constructions}

In this section we introduce our main tools. More precisely, in Section 3.1, given a bounded poset $\mathcal{P}$ and a positive integer $m$, we construct a certain subposet of the direct product $\mathcal{P}^{m}$ and discuss some of its properties. In Section 3.2 we focus on the set of $m$-Dyck paths of height $n$ and introduce a decomposition of them into $m$-tuples of classical Dyck paths of same height.

\subsection{The m-Cover Poset}

Let $\mathcal{P}=\left(P, \leq_{\mathcal{P}}\right)$ be a bounded poset, $m>0$ and consider $m$-tuples of the form

$$
\mathbf{p}=(\underbrace{0_{\mathcal{P}}, 0_{\mathcal{P}}, \ldots, 0_{\mathcal{P}}}_{l_{1}}, \underbrace{p, p, \ldots, p}_{l_{2}}, \underbrace{q, q, \ldots, q}_{l_{3}}),
$$

with $l_{1}+l_{2}+l_{3}=m$. We will usually abbreviate 11 by $\mathbf{p}=\left(0_{\mathcal{P}}^{l_{1}}, p^{l_{2}}, q^{l_{3}}\right)$.

Definition 3.1 Let $\mathcal{P}=\left(P, \leq_{\mathcal{P}}\right)$ be a bounded poset and let $m>0$. Consider the set

$$
P^{\langle m\rangle}=\left\{\left(0^{l_{1}}, p^{l_{2}}, q^{l_{3}}\right) \mid p \lessdot \mathcal{P} q, l_{1}+l_{2}+l_{3}=m\right\} .
$$

The poset $\mathcal{P}^{\langle m\rangle}=\left(P^{\langle m\rangle}, \leq_{\mathcal{P}}\right)$ is called the m-cover poset of $\mathcal{P}$.

We note that in Definition 3.1 we understand $\mathcal{P}^{\langle m\rangle}$ as a subposet of the $m$-fold direct product of $\mathcal{P}$ with itself, and by abuse of notation we use the same symbol for the partial orders of $\mathcal{P}$ and $\mathcal{P}\langle m\rangle$. Furthermore, for every $m>0$, the poset $\mathcal{P}^{\langle m\rangle}$ is isomorphic to a closed interval of $\mathcal{P}^{\langle m+1\rangle}$. For an example of this construction, see for instance Figure 4 . The length and the cardinality of $\mathcal{P}^{\langle m\rangle}$ are given in the following proposition.

Proposition 3.2 Let $\mathcal{P}=\left(P, \leq_{\mathcal{P}}\right)$ be a bounded poset with $n$ elements, c covering relations and a atoms. Then, for $m>0$, we have $\ell\left(\mathcal{P}^{\langle m\rangle}\right)=m \cdot \ell(\mathcal{P})$ and

$$
\left|P^{\langle m\rangle}\right|=(c-a) \cdot\left(\begin{array}{c}
m \\
2
\end{array}\right)+m(n-1)+1
$$


The join- and meet-irreducible elements of $\mathcal{P}$ are related to the join- and meet-irreducible elements of $\mathcal{P}^{\langle m\rangle}$ in the following way.

Proposition 3.3 Let $\mathcal{P}=\left(P, \leq_{\mathcal{P}}\right)$ be a bounded poset with at least two atoms and two coatoms and let $m>0$. Then,

$$
\begin{aligned}
\mathcal{J}\left(\mathcal{P}^{\langle m\rangle}\right) & =\left\{\left(0_{\mathcal{P}}^{l}, p^{m-l}\right) \in P^{\langle m\rangle} \mid p \in \mathcal{J}(\mathcal{P}) \text { and } 0 \leq l<m\right\}, \quad \text { and } \\
\mathcal{M}\left(\mathcal{P}^{\langle m\rangle}\right) & =\left\{\left(p^{l},\left(p^{\star}\right)^{m-l}\right) \in P^{\langle m\rangle} \mid p \in \mathcal{M}(\mathcal{P}) \text { and } 1 \leq l \leq m\right\} .
\end{aligned}
$$

\subsection{The Strip-Decomposition of m-Dyck Paths}

Let $\mathfrak{p} \in \mathcal{D}_{n}^{(m)}$ with associated height-sequence $\mathbf{h}_{\mathfrak{p}}=\left(h_{1}, h_{2}, \ldots, h_{n m}\right)$. For $i \in\{1,2, \ldots, m\}$ we consider the sequences $\mathbf{h}_{i}=\left(h_{i}, h_{i+m}, \ldots, h_{i+(n-1) m}\right)$. It is straightforward to show (using (6) and (7)) that each $\mathbf{h}_{i}$ is in fact the height-sequence of some Dyck path $\mathfrak{q}_{i} \in \mathcal{D}_{n}$. This gives rise to the following definition.

Definition 3.4 Let $\mathfrak{p} \in \mathcal{D}_{n}^{(m)}$ be an $m$-Dyck path with associated height sequence $\mathbf{h}_{\mathfrak{p}}=\left(h_{1}, h_{2}, \ldots, h_{m n}\right)$ and consider the sequence $\delta(\mathfrak{p}):=\left(\mathfrak{q}_{1}, \mathfrak{q}_{2}, \ldots, \mathfrak{q}_{m}\right)$, where for each $i \in\{1,2, \ldots, m\}$ the path $\mathfrak{q}_{i} \in \mathcal{D}_{n}$ is determined by the height sequence $\mathbf{h}_{i}$ from above. We call the sequence $\delta(\mathfrak{p})$ the strip-decomposition of $\mathfrak{p}$.

Remark 3.5 The map $\delta: \mathcal{D}_{n}^{(m)} \rightarrow\left(\mathcal{D}_{n}\right)^{m}$ described in Definition 3.4 is injective.

Example 3.6 We continue with Example 2.1. Since $m=3$, we obtain three sequences

$$
\begin{aligned}
& \mathbf{h}_{\mathfrak{q}_{1}}=\left(h_{1}, h_{4}, h_{7}, h_{10}, h_{13}\right)=(1,3,3,4,5), \\
& \mathbf{h}_{\mathfrak{q}_{2}}=\left(h_{2}, h_{5}, h_{8}, h_{11}, h_{14}\right)=(1,3,3,5,5), \quad \text { and } \\
& \mathbf{h}_{\mathfrak{q}_{3}}=\left(h_{3}, h_{6}, h_{9}, h_{12}, h_{15}\right)=(3,3,4,5,5),
\end{aligned}
$$

which correspond to the three Dyck paths shown in Figure $1(b)$,

\section{The $m$-Cover Poset of the Tamari lattice}

In this section we investigate the $m$-cover poset of $\mathcal{T}_{n}$. In the case $n=3$, Proposition 5.1 implies that $\mathcal{T}_{n}^{\langle m\rangle}$ is always a lattice, while this is no longer true for $n>3$. On the other hand, by combining Proposition 3.2 and [5. Theorem 5.3], we obtain $\left|\mathcal{D}_{n}^{\langle m\rangle}\right|<\mathrm{Cat}^{(m)}(n)$ for $n>3$ and $m>1$. Thus, it makes sense to consider a lattice completion of $\mathcal{T}_{n}^{\langle m\rangle}$, and investigate its relation to $\mathcal{T}_{n}^{(m)}$. And indeed, it turns out that the Dedekind-MacNeille completion of $\mathcal{T}_{n}^{\langle m\rangle}$ (namely the smallest lattice that contains $\mathcal{T}_{n}^{\langle m\rangle}$ as a subposet) is isomorphic to $\mathcal{T}_{n}^{(m)}$. We sketch the proof of this connection in the next section.

\subsection{Proof of Theorem 1.1}

The first step towards the proof of Theorem 1.1 is to show that the strip-decomposition $\delta$, see Definition 3.4 is an order-isomorphism between the restrictions of $\mathcal{T}_{n}^{(m)}$ and $\mathcal{T}_{n}^{\langle m\rangle}$ to their respective sets of join- and meet-irreducible elements. In particular, we have the following propositions. 
Proposition 4.1 For $m, n>0$ the posets $\left(\mathcal{M}\left(\mathcal{T}_{n}^{(m)}\right), \leq_{R}\right)$ and $\left(\mathcal{M}\left(\mathcal{T}_{n}^{\langle m\rangle}\right), \leq_{R}\right)$ are isomorphic.

Proof (sketch): Let $\mathfrak{p} \in \mathcal{M}\left(\mathcal{T}_{n}^{(m)}\right)$. Then, by Proposition 2.2. we have $\mathbf{u}_{\mathfrak{p}}=(0,0, \ldots, 0, a, a, \ldots, a)$, where $a$ first appears at the $(i+1)$-st position and satisfies $a \leq m i$. Moreover, if $a=k m+t$, with $t \in\{0,1, \ldots, m-1\}$, then $\delta(\mathfrak{p})=\left(\mathfrak{q}_{1}, \mathfrak{q}_{2}, \ldots, \mathfrak{q}_{m}\right)$, where

$$
\mathbf{u}_{\mathfrak{q}_{j}}= \begin{cases}(0,0, \ldots, 0, k+1, k+1, \ldots, k+1), & \text { if } j \leq t \\ (0,0, \ldots, 0, k, k, \ldots, k), & \text { if } j>t\end{cases}
$$

and $k+1$ (respectively $k$ ) first appears at the $(i+1)$-st position of $\mathbf{u}_{\mathfrak{q}_{j}}$. Now, showing that $\delta$ is indeed a poset isomorphism is straightforward.

Proposition 4.2 For $m, n>0$ the posets $\left(\mathcal{J}\left(\mathcal{T}_{n}^{(m)}\right), \leq_{R}\right)$ and $\left(\mathcal{J}\left(\mathcal{T}_{n}^{\langle m\rangle}\right), \leq_{R}\right)$ are isomorphic.

Proof (sketch): Let $\mathfrak{p} \in \mathcal{J}\left(\mathcal{T}_{n}^{(m)}\right)$, and let $\delta(\mathfrak{p})=\left(\mathfrak{q}_{1}, \mathfrak{q}_{2}, \ldots, \mathfrak{q}_{m}\right)$. Let $i, k$ and $s$ be the parameters of $\mathfrak{p}$ stated in Proposition 2.3. Then,

$$
\mathbf{u}_{\mathfrak{q}_{j}}= \begin{cases}(0,1, \ldots, n-1), & \text { if } j \leq m-s, \\ (0,1, \ldots, i-2, i-2, i-1 \ldots, k-2, k, k+1 \ldots, n-1), & \text { if } j>m-s .\end{cases}
$$

Again, showing that $\delta$ is a poset isomorphism is straightforward.

Now, the result is implied almost immediately by the following theorem.

Theorem 4.3 ([3] Theorem 7.42]) Let $\mathcal{L}$ be a lattice with no infinite chains. Then,

$$
\mathcal{L} \cong \mathbf{D M}(\mathcal{J}(\mathcal{L}) \cup \mathcal{M}(\mathcal{L})),
$$

where $\mathbf{D M}$ denotes the Dedekind-MacNeille completion.

Proof of Theorem 1.1 (Sketch): First, we remark that every element in $\mathcal{D}_{n}^{\langle m\rangle}$ can be expressed as the join of elements in $\mathcal{J}\left(\mathcal{T}_{n}^{\langle m\rangle}\right)$. Thus $\boldsymbol{D M}\left(\mathcal{J}\left(\mathcal{T}_{n}^{\langle m\rangle}\right) \cup \mathcal{M}\left(\mathcal{T}_{n}^{\langle m\rangle}\right)\right) \cong \boldsymbol{D M}\left(\mathcal{T}_{n}^{\langle m\rangle}\right)$. The application of this isomorphism and Propositions 4.1 and 4.2 to Theorem 4.3 yields

$$
\mathcal{T}_{n}^{(m)} \cong \boldsymbol{D M}\left(\mathcal{J}\left(\mathcal{T}_{n}^{(m)}\right) \cup \mathcal{M}\left(\mathcal{T}_{n}^{(m)}\right)\right) \cong \boldsymbol{D M}\left(\mathcal{J}\left(\mathcal{T}_{n}^{\langle m\rangle}\right) \cup \mathcal{M}\left(\mathcal{T}_{n}^{\langle m\rangle}\right)\right) \cong \boldsymbol{D M}\left(\mathcal{T}_{n}^{\langle m\rangle}\right)
$$

Example 4.4 Figure 3 shows the Dedekind-MacNeille completion of $\mathcal{T}_{4}^{\langle 2\rangle}$, which is indeed isomorphic to $\mathcal{T}_{4}^{(2)}$. The orange elements are not contained in $\mathcal{D}_{4}^{\langle 2\rangle}$. The following remark indicates that there is yet another explanation for the orange elements: they are a certain modification of the strip-decomposition of the corresponding m-Dyck paths. 


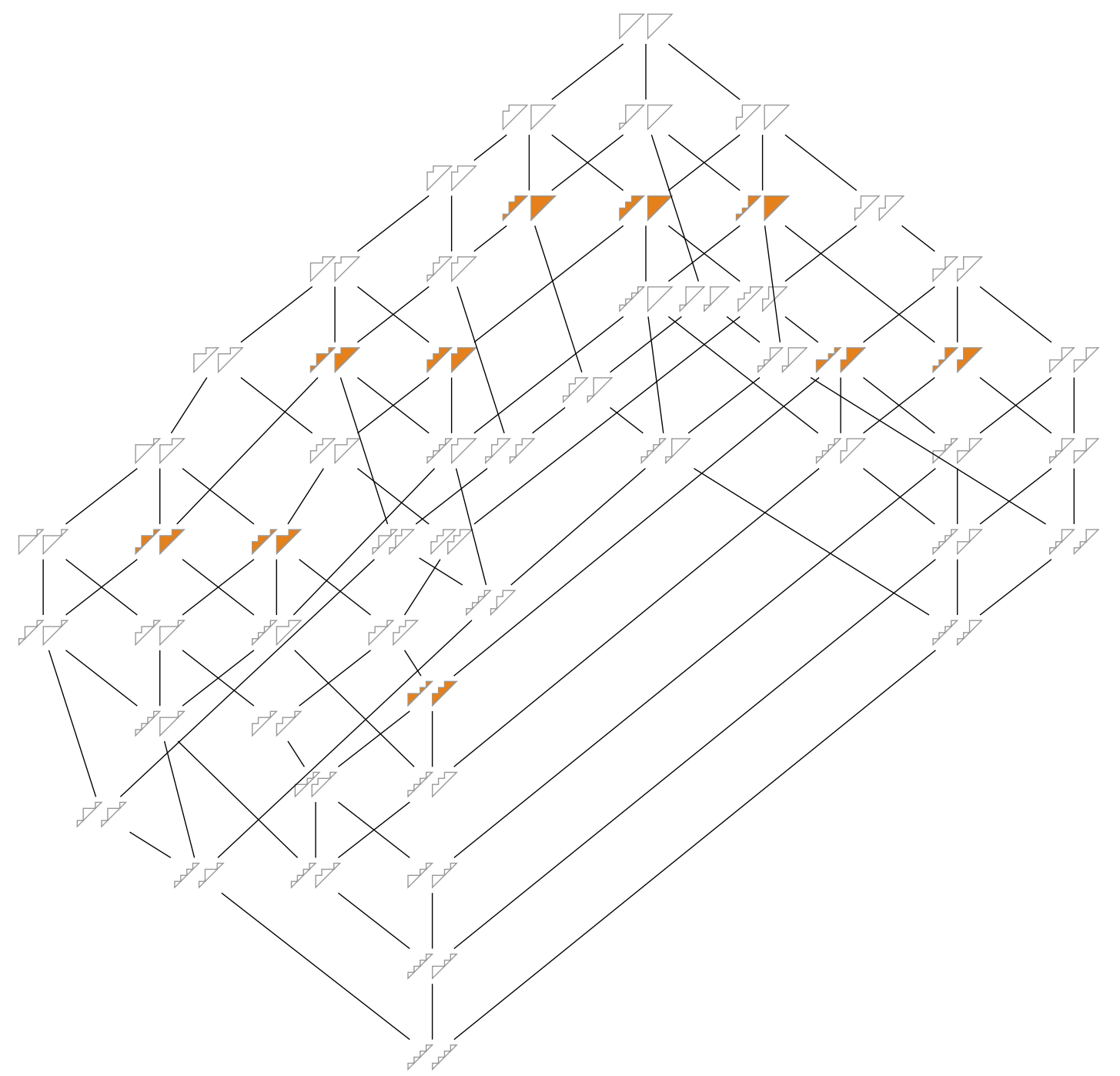

Fig. 3: The lattice $\boldsymbol{D M}\left(\mathcal{T}_{4}^{\langle 2\rangle}\right)$. The orange elements are added during the lattice completion. 
Remark 4.5 Consider the map $\beta_{i, j}:\left(\mathcal{D}_{n}\right)^{m} \rightarrow\left(\mathcal{D}_{n}\right)^{m}$ defined by

$$
\beta_{i, j}\left(\mathfrak{q}_{1}, \ldots, \mathfrak{q}_{m}\right)=\left(\mathfrak{q}_{1}, \ldots, \mathfrak{q}_{i-1}, \mathfrak{q}_{i} \wedge_{R} \mathfrak{q}_{j}, \mathfrak{q}_{i+1}, \ldots \mathfrak{q}_{j-1}, \mathfrak{q}_{i} \vee_{R} \mathfrak{q}_{j}, \mathfrak{q}_{j+1}, \ldots, \mathfrak{q}_{m}\right),
$$

and define the bouncing map to be the composition $\beta=\beta_{m-1, m} \circ \cdots \circ \beta_{2,3} \circ \beta_{1, m} \circ \cdots \circ \beta_{1,2}$, acting from the left. Then, computer experiments suggest the following, surprising connection:

Conjecture 4.6 For $m, n>0$, we have $\left(\mathcal{D}_{n}^{(m)}, \leq_{R}\right) \cong\left(\beta \circ \delta\left(\mathcal{D}_{n}^{(m)}\right), \leq_{R}\right)$.

\section{The $m$-Cover Posets which are Lattices}

In the last section of this abstract we focus on the $m$-cover posets and sketch the proofs of Theorem 1.2 and Theorem 1.3 .

\subsection{Proof of Theorem 1.2}

By distinguishing cases in one direction and arguing by contradiction in the other direction, it is not hard to prove the following characterization.

Proposition 5.1 Let $\mathcal{P}=\left(P, \leq_{\mathcal{P}}\right)$ be a bounded poset, and let $m>1$. Then, $\mathcal{P}^{\langle m\rangle}$ is a lattice if and only if $\mathcal{P}$ is a lattice and for every $x, y \in P$ we have $x \wedge_{\mathcal{P}} y \in\left\{0_{\mathcal{P}}, x, y\right\}$.

Proof of Theorem 1.2 ; Let $H$ denote Hasse diagram of $\mathcal{P}$ with $0_{\mathcal{P}}$ removed, and let $x, y \in P$.

If $H$ is a tree, then it does not contain a cycle, and it is straightforward to verify that $\mathcal{P}$ is a lattice and that the meet of any two incomparable elements is the least element. Conversely, suppose that $\mathcal{P}\langle m\rangle$ is a lattice, and thus Proposition 5.1 implies that $\mathcal{P}$ is a lattice such that $p \wedge_{\mathcal{P}} q \in\left\{p, q, 0_{\mathcal{P}}\right\}$ for all $p, q \in P$. Suppose that $H$ is not a tree, and must thus contain a cycle. Then, we can find elements $p, q, z \in H$ in this cycle such that $z \leq_{\mathcal{P}} p, q$, which implies $z \leq_{\mathcal{P}} p \wedge_{\mathcal{P}} q$. By assumption, we obtain $z=0_{\mathcal{P}}$ contradicting $z \in H$. Hence, $H$ is a tree, and the proof is completed.

\subsection{Proof of Theorem 1.3}

Let now $k>1$, and let $\mathfrak{D}_{k}$ denote the dihedral group of order $2 k$, and let $\mathcal{C}_{k}=\left(C_{k}, \leq_{k}\right)$ denote the Cambrian lattice associated with $\mathfrak{D}_{k}$, see [9]. We remark that $\mathcal{C}_{k}$ with least and greatest element removed, is in fact a disjoint union of a chain having $k-1$ elements, say $c_{1} \lessdot_{k} c_{2} \lessdot_{k} \cdots \lessdot_{k} c_{k-1}$, and a singleton, say $a_{1}$.

Before we proceed to the sketch of the proof of Theorem 1.3 , we recall the following result.

Theorem 5.2 ([12, Theorem 7]) Let $\mathcal{L}$ be a finite lattice. If $\mathcal{L}$ is trim and nuclear, then its order complex is homotopic to a sphere, whose dimension is 2 less than the number of atoms of $\mathcal{L}$. If $\mathcal{L}$ is trim but not nuclear, then its order complex is contractible.

Proof of Theorem 1.3 (Sketch): First of all, notice that it follows directly from Theorem 1.2 and Proposition 3.2 that $\mathcal{C}_{k}^{\langle m\rangle}$ is a lattice with cardinality equal to $\left(\begin{array}{c}m+1 \\ 2\end{array}\right) k+m+1$. We will prove now that in addition it is trim. To start, we show that $\mathcal{C}_{k}^{\langle m\rangle}$ is extremal. Indeed, consider the following maximal chain:

$$
c:\left(0^{m}\right) \lessdot\left(0^{m-1}, c_{1}\right) \lessdot \cdots\left(c_{1}^{m}\right) \lessdot\left(c_{1}^{m-1}, c_{2}\right) \lessdot \cdots\left(c_{k-1}^{m-1}, 1\right) \lessdot\left(1^{m}\right)
$$




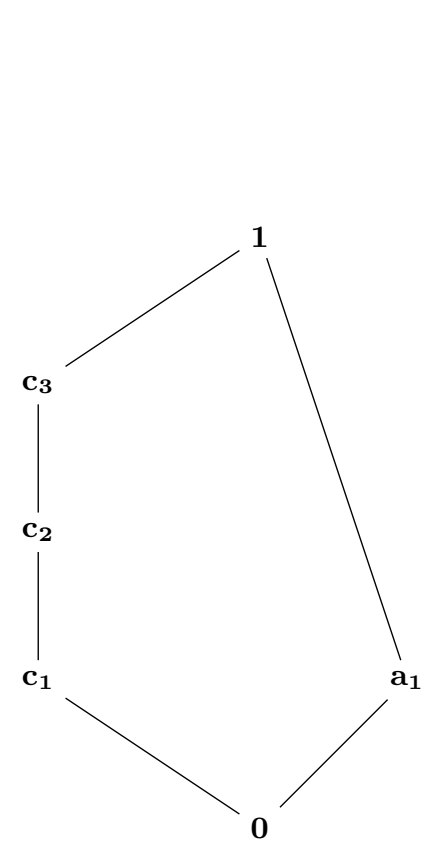

(a) The lattice $\mathcal{C}_{4}$.

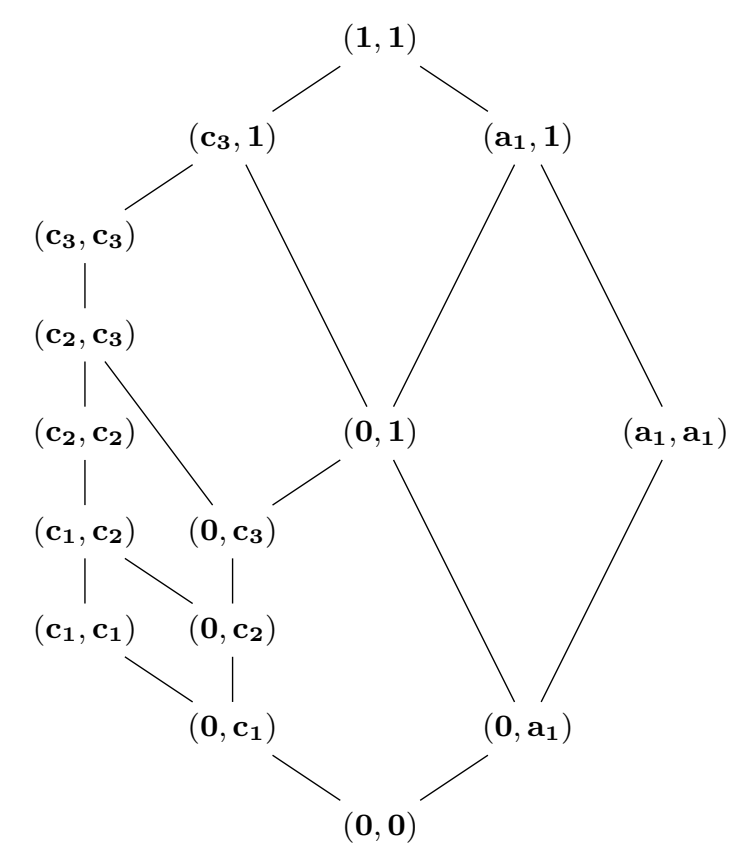

(b) The lattice $\mathcal{C}_{4}^{\langle 2\rangle}$.

Fig. 4: Illustration of the $m$-cover construction.

and remark that it is a chain having maximum length, equal to $k m$. Thus, $\ell\left(\mathcal{C}_{k}^{\langle m\rangle}\right)=k m$. On the other hand, it follows easily from Proposition 3.3 that $\mathcal{J}\left(\mathcal{C}_{k}^{\langle m\rangle}\right)=\mathcal{M}\left(\mathcal{C}_{k}^{\langle m\rangle}\right)=k m$ as well. This implies that $\mathcal{C}_{k}^{\langle m\rangle}$ is extremal. Now, we consider again the maximal chain $c$ and remark that it consists of left-modular elements. It follows therefore that $\mathcal{C}_{k}^{\langle m\rangle}$ is trim.

Finally, it is straightforward to show that every element in $\mathcal{C}_{k}^{\langle m\rangle}$ has at most two upper covers and at most two lower covers. Thus, if $\left[\mathbf{x}, \mathbf{x}^{\prime}\right]$ is nuclear, then we have either $\mathbf{x} \lessdot_{k} \mathbf{x}^{\prime}$ or $\mathbf{x}^{\prime}$ is the join of the two atoms in $\left[\mathbf{x}, \mathbf{x}^{\prime}\right]$, and hence we obtain $\mu\left(\mathbf{x}, \mathbf{x}^{\prime}\right)= \pm 1$ as desired. If $\left[\mathbf{x}, \mathbf{x}^{\prime}\right]$ is not nuclear, then Theorem 5.2 implies that the associated order complex is contractible, and hence has reduced Euler characteristic 0. Proposition 3.8.6 in [10] implies that the Möbius function of an interval in a poset takes the same value as the reduced Euler characteristic of the associated order complex, and the result follows.

Example 5.3 Consider the dihedral group $\mathfrak{D}_{4}$, and let $m=2$. The corresponding lattice $\mathcal{C}_{4}$ is illustrated in Figure $4(a)$ The elements of $C_{4}^{\langle 2\rangle}$ are precisely

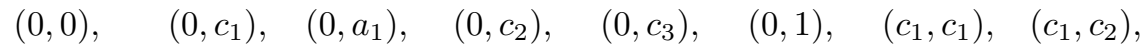

$$
\begin{aligned}
& \begin{array}{llllll}
\left(a_{1}, a_{1}\right), & \left(a_{1}, 1\right), \quad\left(c_{2}, c_{2}\right), & \left(c_{2}, c_{3}\right), & \left(c_{3}, c_{3}\right), & \left(c_{3}, 1\right), & (1,1),
\end{array}
\end{aligned}
$$

and the lattice $\mathcal{C}_{4}^{\langle 2\rangle}$ is illustrated in Figure $4(b)$ 
We complete our investigation of the lattice $\mathcal{C}_{k}^{\langle m\rangle}$ with the study of a certain statistic. Given a poset $\mathcal{P}=\left(P, \leq_{\mathcal{P}}\right)$, denote by $u_{n}$ the number of elements in $P$ having precisely $n$ upper covers, and consider the generating function $\operatorname{UF}(x ; \mathcal{P})=\sum_{n \geq 0} u_{n} x^{n}$. Analogously, denote by $l_{n}$ the number of elements in $P$ having precisely $n$ lower covers, and consider the generating function $\operatorname{LF}(x ; \mathcal{P})=\sum_{n \geq 0} l_{n} x^{n}$. We have the following result.

Proposition 5.4 For every $k, m>0$, we have

$$
U F\left(x ; \mathcal{C}_{k}^{\langle m\rangle}\right)=1+k m x+\left(m+k\left(\begin{array}{c}
m \\
2
\end{array}\right)\right) x^{2}=L F\left(x ; \mathcal{C}_{k}^{\langle m\rangle}\right)
$$

Proof: Recall that every element of $\mathcal{C}_{k}^{\langle m\rangle}$ has at most two upper covers, and at most two lower covers. Now, Propositions 3.2 and 3.3 imply the result.

We complete this abstract with the following nice property.

Proposition 5.5 If $k=3$, then $\mathcal{C}_{3}^{\langle m\rangle} \cong \mathcal{T}_{3}^{(m)}$ for all $m>0$.

Proof: It is straightforward to verify that $\mathcal{T}_{3} \cong \mathcal{C}_{3}$. Thus, together with Theorems 1.1 and 1.2 , we obtain

$$
\mathcal{C}_{3}^{\langle m\rangle} \cong \mathcal{T}_{3}^{\langle m\rangle} \cong \boldsymbol{D M}\left(\mathcal{T}_{3}^{\langle m\rangle}\right) \cong \mathcal{T}_{3}^{(m)}
$$

\section{References}

[1] F. Bergeron and L.-F. Préville-Ratelle, Higher Trivariate Diagonal Harmonics via Generalized Tamari Posets, J. Combinatorics 3 (2012), 317-341.

[2] M. Bousquet-Mélou, É. Fusy, and L.-F. Préville-Ratelle, The Number of Intervals in the m-Tamari Lattices, Elec. J. Combin. 18 (2011).

[3] B. A. Davey and H. A. Priestley, Introduction to Lattices and Order, Cambridge University Press, Cambridge, 2002.

[4] P. Duchon, On the Enumeration and Generation of Generalized Dyck Words, Discrete Math. 225 (2000), $121-135$.

[5] W. Geyer, On Tamari Lattices, Discrete Math. 133 (1994), 99-122.

[6] M. Kallipoliti and H. Mühle, The m-Cover Posets and the Strip-Decomposition of m-Dyck Paths, arXiv:1312.2520 (2013).

[7] G. Markowsky, Primes, Irreducibles and Extremal Lattices, Order 9 (1992), 265-290.

[8] N. Reading, Cambrian Lattices, Adv. Math. 205 (2006), 313-353.

[9] N. Reading, Sortable Elements and Cambrian Lattices, Algebra Univ. 56 (2007), 411-437.

[10] R. P. Stanley, Enumerative Combinatorics, Vol. 1, Cambridge University Press, Cambridge, 1997.

[11] D. Tamari, The Algebra of Bracketings and their Enumeration, Nieuw Archief voor Wiskunde 10 (1962), $131-146$.

[12] H. Thomas, An Analogue of Distributivity for Ungraded Lattices, Order 23 (2006), 249-269. 